\title{
Emotional profiling through supervised machine learning of interrupted EEG interpolation
}

\author{
Hamwira Yaacob $^{1^{*}}$, Hazim Omar ${ }^{2}$, Dini Handayani ${ }^{3}$ and Raini Hassan ${ }^{1}$ \\ Assistant Professor, International Islamic University Malaysia, Malaysia ${ }^{1}$ \\ PhD Student, Universiti Sains Malaysia, Malaysia $^{2}$ \\ Lecturer, Taylor's University, Malaysia ${ }^{3}$
}

Received: 31-August-2018; Revised: 16-January-2019; Accepted: 30-January-2019

(C)2019 Hamwira Yaacob et al. This is an open access article distributed under the Creative Commons Attribution (CC BY) License, which permits unrestricted use, distribution, and reproduction in any medium, provided the original work is properly cited.

\begin{abstract}
It has been reported that the construction of emotion profiling models using supervised machine learning involves data acquisition, signal pre-processing, feature extraction and classification. However, almost all papers do not address the issue of profiling emotion using supervised machine learning on the interrupted encephalogram (EEG) signals. Based on a preliminary study, emotion profiling on interrupted EEG signals produces low classification accuracy, using multilayer perceptron (MLP). Furthermore, lower emotion classification accuracy is produced from interrupted EEG signals with higher number of segments. Thus, the objective of this paper is to propose a technique and present the outcomes of handling interrupted EEG signals for emotion profiling. This is done by the suppression and interpolation of originally interrupted EEG signals at pre-process stage. As a result, emotion classification using MLP on interpolated data improves from $80.1 \%$ to $95 \%$.
\end{abstract}

\section{Keywords}

Interrupted EEG, Interpolation, Emotion classification, Power spectral density.

\section{Introduction}

Several computational models have been proposed to determine the states of emotion from brain signals, which can be captured using different modalities, including electroencephalogram (EEG). Through EEG, electrical signals that are emancipated during brain activation are amplified and recorded. In most studies, construction of the computational models for emotional state classification using EEG involves data acquisition, signal preprocessing, feature extraction and training. During data collection, EEG signals are recorded while brain stimulation tasks are performed. Unwanted noise and artifacts are filtered through pre-processing step. Consequently, features as the input for the classification through supervised machine learning are extracted from the filtered signals. Different set of features has been experimented including statistical features [1, 2], Mel-frequency Cepstral coefficients [3], Kerneldensity Estimation [4], Gabor visual features [5] and CMAC-based model of the effects [6].

\footnotetext{
*Author for correspondence
}

However, the most fundamental features, which is also the most commonly used features are the power spectral density [7-11].

Likewise, different machine learning models have been employed to perform emotion classification, including multilayer perceptron [3], [12-14], support vector machine [1, 15], k-nearest neighbor [16], adaptive Neuro-fuzzy inference systems [5], AdaBoost [17], dynamical graph convolutional neural network [18] and many others.

However, almost all papers do not address the issue of profiling emotion using supervised machine learning on interrupted EEG signals, especially recorded by wireless EEG machines due to unstable connectivity issues. Based on a preliminary study, emotion profiling on interrupted EEG signals produces low classification accuracy.

Therefore, the objective of this paper is to propose a technique to handle interrupted EEG signals for emotion classification. Related works are presented in the following section, which is succeeded by the 
methods. Results of emotion classification using interrupted EEG and interpolated data are presented before the Conclusion.

\section{Related works}

Based on the current states of the art, profiling of emotion through machine learning involves data acquisition, preprocessing and supervised machine learning. Data acquisition includes the recording of EEG signals during brain stimulation tasks. Through preprocessing, unwanted are filtered out and features are extracted. Fundamentally, power spectral density (PSD) is widely used as the primary features for emotion classification using EEG signals, hence it is discussed below. Furthermore, many supervised machine learning classifiers are used to perform emotion classification, as elaborated in this section. As indicated in [19], emotion classification is performed through different approaches of emotion stimulation during EEG signal recording, different feature extraction techniques and different classification algorithms.

For emotion stimulation, photographs from the international affective picture standard (IAPS) [20] dataset are widely used as stimuli set. Each photograph has been rated based on valence, arousal and dominance, which then become the reference for the selection of stimuli set. For example, in a study [21], the measurements of valence and arousal are used to select and classify IAPS photographs that are then presented to the subjects for emotion stimulation while EEG signal are recorded. Based on valence and arousal, IAPS photographs are also categorized as either calm, positively excited and negative excited emotional states [22]. In other studies, different categories of emotional stimuli are derived such as calm-neutral and negatively excited [23, 24], happiness, sadness, fear and calm [3], and many others.

Furthermore, many approaches have been implemented to extract features from recorded EEG signals for emotion classification. Different approaches include the number of EEG electrodes, frequency bands, filtering and feature extraction techniques are implemented. In terms of the number of electrodes, it is inferred from the state of the art that the standard number of EEG electrodes for emotion classification has not been established yet. Among others, the numbers of electrodes analysed for emotion classification are 64 electrodes [25, 26], 14 electrodes [15, 18], 8 electrodes $[3,27]$ and 5 electrodes [17, 28].
Although various techniques have been implemented to extract features from EEG signals for emotion classification, including statistical features $[1,2]$, Mel-frequency Cepstral coefficients [3, 12], Kerneldensity Estimation [4], Gabor visual features [5] and CMAC-based Model of Affects [6], power spectral density [29] being the most fundamental technique for extracting features is still used [7-11].

To perform emotion classification, different machine learning classifiers have been employed. Using multilayer perceptron (MLP) as the classifier to classify happy, love, sad and anger, an average of $78.11 \%$ accuracy was obtained [13]. In another study, MLP was used to measure valence and arousal for classifying happy, sad, fear and calm [12]. In the study, emotion classifiers are further applied to analyze the stress level of primary school teachers. In addition to that, MLP is used to classify emotion based on recalibrated speech affective space model (rSASM) and 12-point affective circumplex (12PAC) [3]. At an average, classifying emotion using MLP based on rSASM produced an accuracy of $78.5 \%$, which is $14.5 \%$ lower than the accuracy on 12-PAC model.

In other words, different techniques and algorithms have been implemented for classifying emotion from EEG signals. However, almost all papers do not address the issue of profiling emotion using supervised machine learning on the interrupted encephalogram (EEG) signals. In this paper, the concern is elaborated and a solution is proposed.

\section{Methods}

This study was implemented through several processes, including data acquisition, pre-processing and supervised machine learning:

\section{Data Acquisition}

Data was obtained from Universiti Sains Malaysia (USM) through neural plasticity, functional reorganization and network reconfiguration of the human brain after traumatic brain injury in cognitive, language and attention processing study. Data acquisition involves the recording of EEG signals during brain stimulation, which is executed after obtaining a written informed consent from the participants. In this paper, only EEG recordings of 6 participants are analyzed and reported. EEG signals are recorded using 64 channels A.N.T. EegoSport based on the international standard of 10-20 EEG electrode position [30]. With the sampling rate of $1000 \mathrm{~Hz}$ and reference at $\mathrm{CPz}$, the channels are $\mathrm{Fp} 1$, 
Fpz, Fp2, F7, F3, Fz, F4, F8, FC5, FC1, FC2, FC6, M1, T7, C3, Cz, C4, T8, M2, CP5, CP1, CP2, CP6, P7, P3, Pz, P4, P8, POz, O1, O2, EOG, AF7, AF3, AF4, AF8, F5, F1, F2, F6, FC3, FCz, FC4, C5, C1, C2, C6, CP3, CP4, P5, P1, P2, P6, PO5, PO3, PO4, PO6, FT7, FT8, TP7, TP8, PO7, PO8 and Oz.

The recording of EEG signal intends to capture two major mental states, namely resting state and emotional state. Each resting state is recorded for 30 seconds, while each emotional state is recorded for
60 seconds. As depicted in Figure 1, the recording of EEG starts with a resting state, which is set in between two emotional states. The sequence of emotional state is sadness, calm, happiness and fear. Thus, the entire protocol is completed in 400 seconds. During the resting state, participants are at idle. On the other hand, during the emotional states, participants are expected to observe the corresponding emotional stimuli presentation.

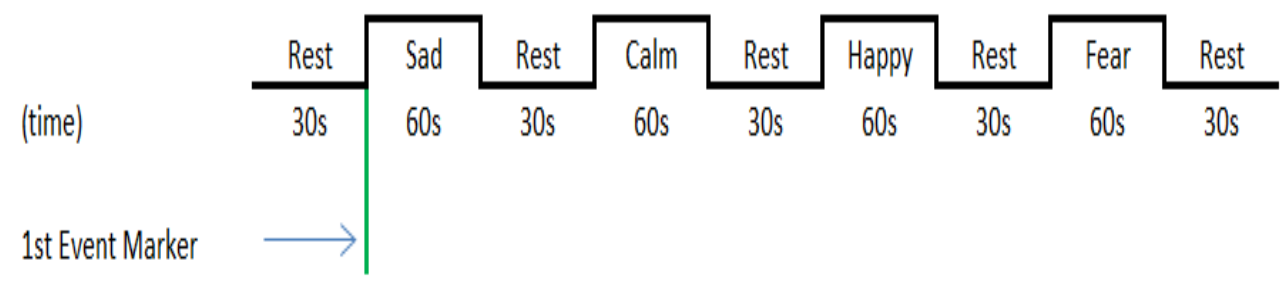

Figure 1 EEG recording protocol

Emotional stimuli are obtained from the International Affective Picture System (IAPS) that provides normative ratings of emotion for a set of color photographs [20]. Each photo-graph has been rated based on valence, arousal and dominance. In this paper, only valence and arousal are analyzed. Therefore, a 2-dimensional affective space [31] that comprises of valence and arousal as the horizontal and vertical axes is referred.

As illustrated in Figure 2, emotional states that are located on the right of the vertical axis are considered as having a positive valence, and emotional states that are located on the left of the vertical axis are considered as having a negative valence. Likewise, emotional states that are located on top of the horizontal axis are considered as having positive arousal, and emotional states that are located below the horizontal are considered as having negative arousal. Furthermore, emotional states with positive valence and positive arousal are labelled as Happiness, emotional states with negative valence and positive arousal are labelled as Fear, emotional states with negative valence and negative arousal are labelled as Sadness and emotional states with positive valence and negative arousal are labelled as Calm.

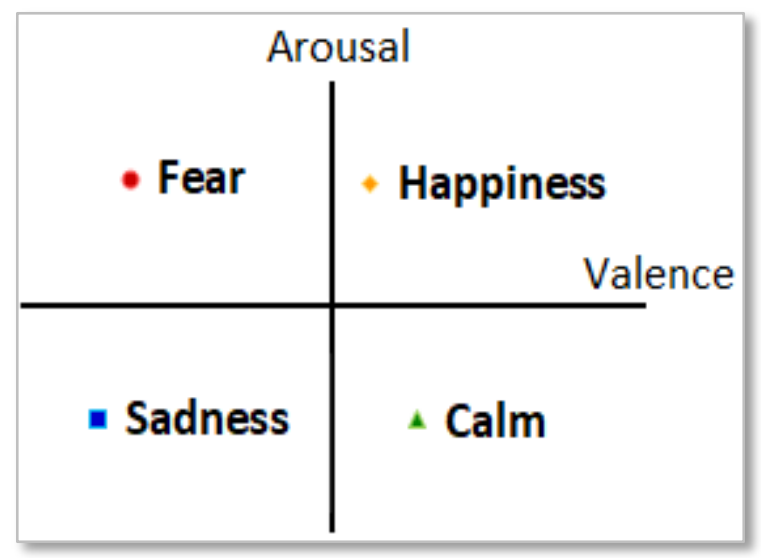

Figure 2 Affective space model

The selection of emotional stimuli is performed based on the ranking of normative ratings, where photographs at the higher ranks are categorized as positive, and photographs at the lower ranks are categorized as negative. Nevertheless, photographs with extreme objects and scenes such as mutilated bodies and erotic nudes are excluded. With that, 6 sets of each emotional stimuli are extracted. Each participant is presented with only one set for each emotional state. Table 1 shows the ID of photographs in IAPS which are grouped into different emotional stimuli sets. 
Table 1 Emotion stimuli ID

\begin{tabular}{|c|c|}
\hline Set & Photograph ID \\
\hline Calm 1 & $1610,2035,2370,5010,5200,5551,5725,5779,5811,7325$ \\
\hline Calm 2 & $1604,1620,2304,2360,2598,5000,5202,5760,5780,5891$ \\
\hline Calm 3 & $2211,4561,5300,5395,5870,5891,5991,7500,7550,7820$ \\
\hline Calm 4 & $2211,4561,5300,5395,5870,5891,5991,7500,7550,7820$ \\
\hline Calm 5 & $1410,1450,1500,1510,1600,1601,1602,1603,1604,1620$ \\
\hline Calm 6 & $1122,1460,1616,1740,1812,1850,1903,1942,2019,2020$ \\
\hline Fear 1 & $3030,3053,3060,3080,3120,3170,3266,6563,9410,9413$ \\
\hline Fear 2 & $3000,3010,3068,3069,3071,3130,3530,6313,6350,9940$ \\
\hline Fear 3 & $3001,3064,3170,3500,6230,6231,6250.1,6250,9600,9940$ \\
\hline Fear 4 & $1114,1525,1932,2811,3068,3069,6315,8475,8485,9635.1$, \\
\hline Fear 5 & $1019,1022,1030,1033,1040,1051,1070,1080,1090,1101$ \\
\hline Fear 6 & $1050,1052,1300,1304,1321,1930,2053,2352.2,2800,2900$ \\
\hline Happiness 1 & $5629,7405,8034,8163,8185,8190,8200,8300,8400,8492$, \\
\hline Happiness 2 & $5470,5621,8030,8080,8170,8186,8370,8490,8499,8501$, \\
\hline Happiness 3 & $1121,1313,1340,1350,1440,1463,1540,1560,1595,1630$ \\
\hline Happiness 4 & $4626,8178,8179,8186,8191,8192,8193,8251,8341,8499$ \\
\hline Happiness 5 & $1340,1463,1811,1999,2040,2045,2050,2057,2058,2070$ \\
\hline Happiness 6 & $2018,2122,2220,2616,4505,4525,4575,4598,4610,4628$ \\
\hline Sadness 1 & $2301,2455,2900.1,3300,9041,9280,9290,9291,9331,9831$, \\
\hline Sadness 2 & $2205,2276,2456,2750,9000,9220,9330,9342,9561,9832$ \\
\hline Sadness 3 & $2141,2205,2681,5970,6000,9000,9001,9220,9417,9421$ \\
\hline Sadness 4 & 2055.1, 2375.1, 2694, 2716, 2780, 2799, 6311, 9171, 9435, 9926, \\
\hline Sadness 5 & $1112,1270,1275,1945,2002,2026,2101,2130,2190,2200$ \\
\hline Sadness 6 & $1240,1505,2110,2115,2141,2205,2206,2221,2230,2276$ \\
\hline
\end{tabular}

\section{Preprocessing and feature extractions}

It has been observed that the recording of EEG signals using 64 channels A.N.T. EegoSport over an intended duration may be interrupted several times. Therefore, instead of having the entire duration stored as one segment, the signals may be stored in several segments. Table 2 shows the number of segments in EEG recording, which is intended for 400 seconds, for each participant in this study.

Based on the table, only EEG recording on Participant 6 is recorded entirely within one segment, with the total number of 405999 instances. With the total number of 393886 samples, EEG signals of Participant 1 were recorded in 2 segments. This is because the re-cording was interrupted once throughout the intended recording duration. EEG signals of Participant 2 were recorded in 5 segments with a total number of 396946 samples. That is 4 interruptions. EEG signals on Participant 3, Participant 4 and Participant 6 were recorded more than 10 segments.

To illustrate further, Figure 3 shows the time-domain plot of EEG recording on channel Fpz-1 for Participant 1 where EEG signals are recorded with 2 segments. As illustrated, a few seconds of each segment are noise. In this paper, it is generalized that the noise is 5000 samples, that is 5 seconds. The noise is first considered as "no signal" before interpolation of the segment. The results of interpolation on the same channel are displayed in Figure 4. 
Hamwira Yaacob et al.

Table 2 Number of segments in EEG recording

\begin{tabular}{lll}
\hline Participant ID & Number of segments & Total number of instances \\
\hline 1 & 2 & 393886 \\
2 & 5 & 396946 \\
3 & 19 & 396911 \\
4 & 13 & 386556 \\
5 & 16 & 395361 \\
6 & 1 & 405999 \\
\hline
\end{tabular}

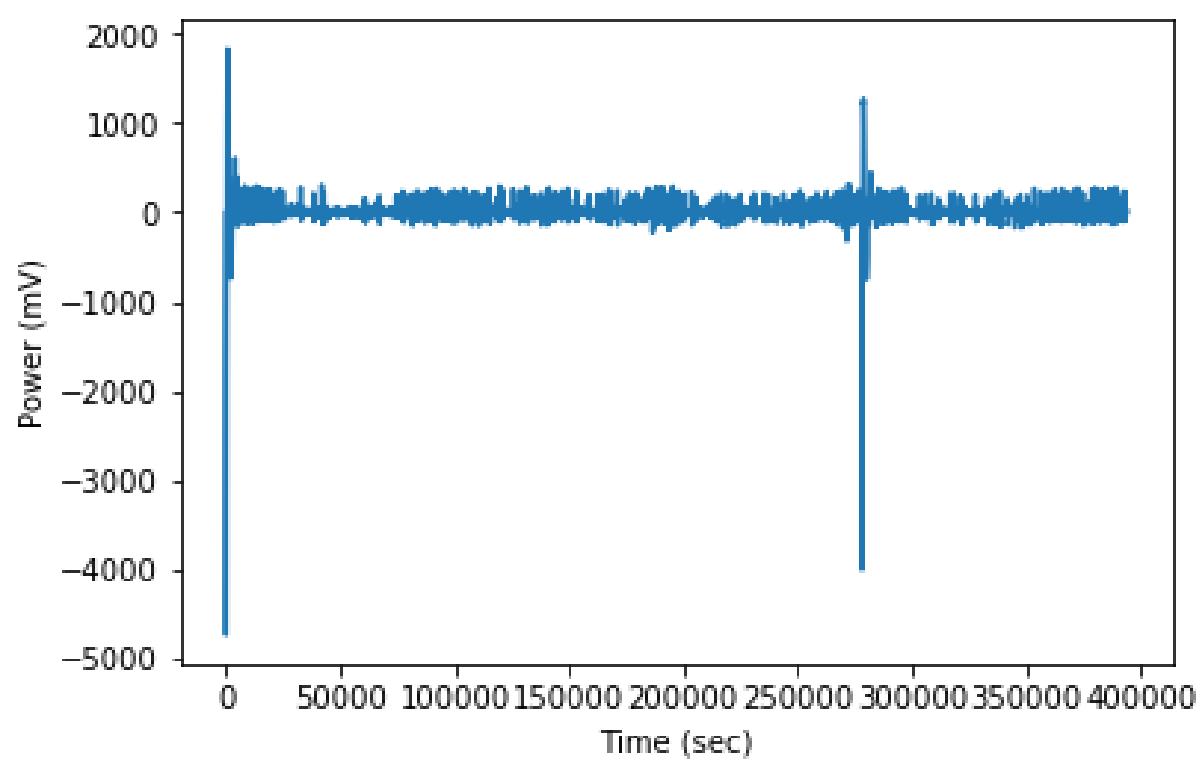

Figure 3 Time-domain plot of EEG recording on channel Fpz-1 for participant 1 before interpolation

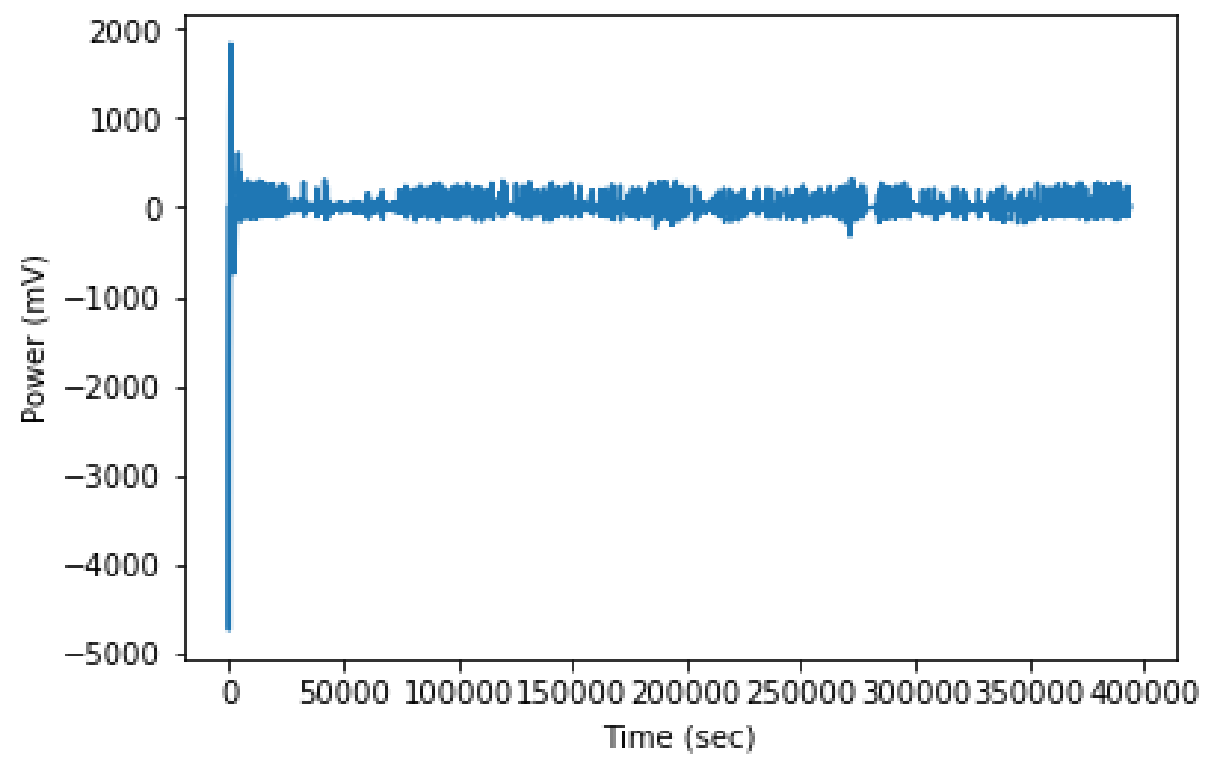

Figure 4 Time-domain plot of EEG recording on channel Fpz-1 for participant 1 after interpolation 
Consequently, interpolated signals are segmented based on the protocol shown in Figure 2. This is done by keeping 360000 instances of interpolated signals from the last (i.e.: Some early instances are discarded). From 360000 instances, the first 60000 instances are labeled as sad, followed by 30000 instances of resting state, then the next 60000 instances are labeled as calm, followed by 30000 instances of resting state, 60000 instances of happy, 30000 instances of resting state, 60000 instances of fear and 30000 instances of resting state.

Next, the power spectral density (PSD) of each segment for each channel on each subject is calculated separately. PSD is calculated by taking the average of spectral power using short-time Fourier transform (STFT). Thus, 64 features correspond to each EEG channel are extracted as the input features.

\section{Supervised learning}

The objective of supervised machine learning in this study is to perform classification of emotional states from a set of input features that are derived from EEG signals. For that purpose, each instance comprises of 64 features (correspond to the PSD of each EEG channel) and 2 target values (correspond to the valence and arousal of the stimuli that are presented during EEG signals are recorded).

Thus, two multilayer perceptron models that consist of 64 input nodes and 1 output node are employed. Each model represents valence and arousal, respectively. For positive valence (or arousal) instances are trained to 1 , while negative instances are trained to 0 . Thus, 0.5 is considered as the threshold for both MLP models. The performance of these models is measured based on the accuracy. The preprocessing and feature extraction have resulted in a total of 1068 instances, in which 267 instances are derived from each of the EEG recordings during happiness, fear, sadness and calm stimulations.

\section{Results}

Emotion classification is measured based on the classification of valence and the classification of arousal. Thus, the output is presented in a fourquadrant affective space, where the horizontal axis represents valence and the vertical axis represents arousal.

\subsection{Emotion classification of original data}

The result for emotion classification of original data for each participant is presented in Figure 5. The accuracy of emotion classification for Participant 1 based on valence is $97 \%$. For Participant 2, the accuracy for classifying valence is $89.4 \%$. From the plot, the distribution of outputs with positive arousal is mostly located towards the axis. Classification accuracy of valence for Participant 3 is $75.1 \%$. Most of the outputs with negative valence are also categorized as negative arousal. On the other hand, most of the outputs with positive valence are overlapped as positive arousal. For Participant 4, the instances mostly fall under the fear quadrant with the accuracy of classifying valence about $64 \%$. The accuracy of classifying interrupted EEG based on valence is $50 \%$ for Participant 5. With only one segment, the classification of valence on Participant 6 is around $99 \%$.
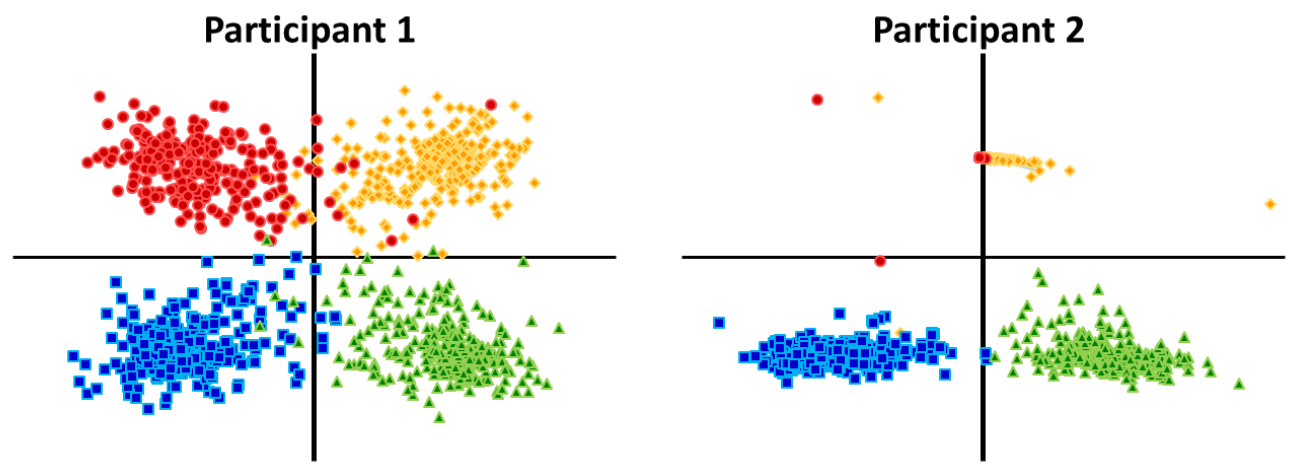

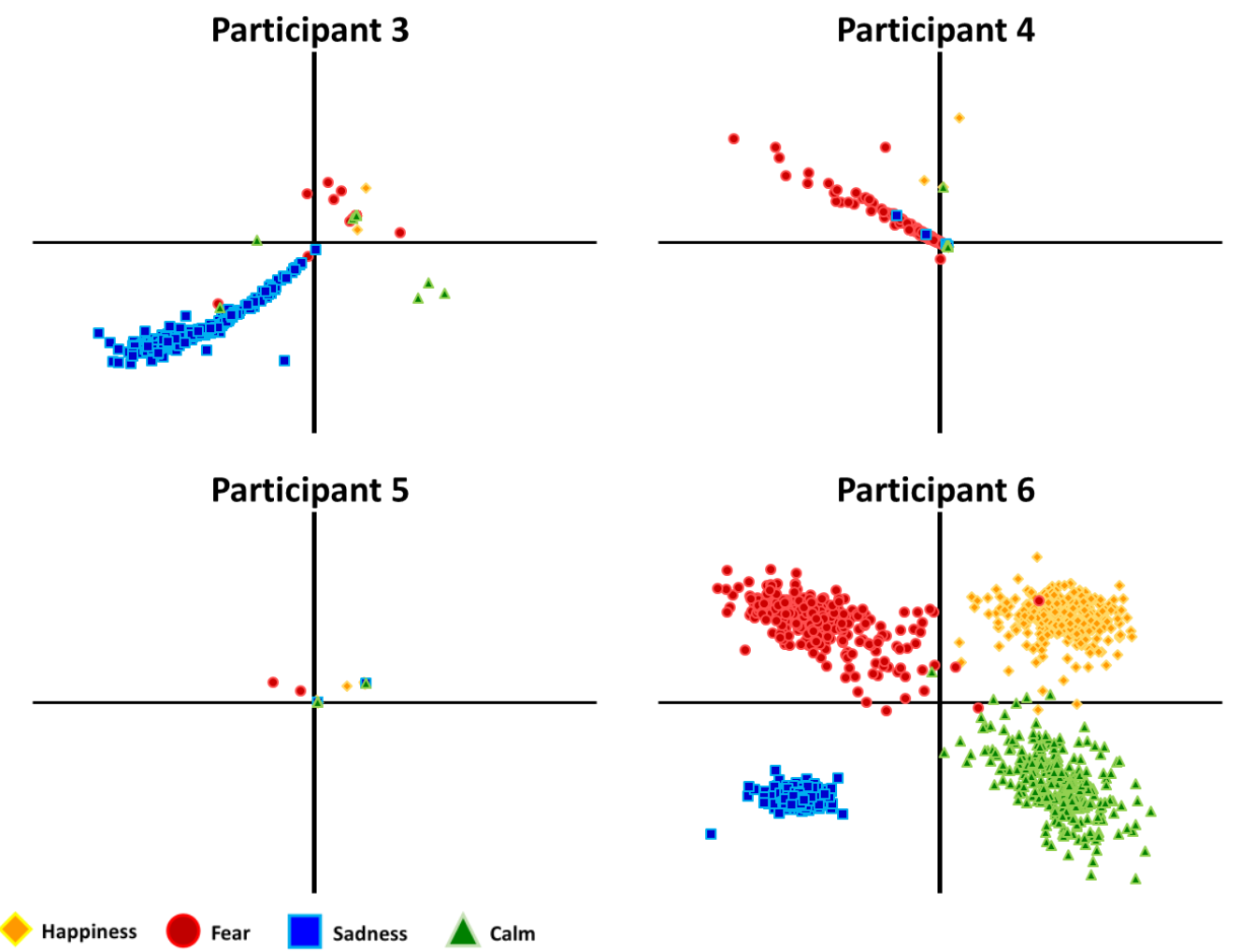

Figure 5 Output of MLP uses original interrupted EEG data on affective space model

For arousal, emotion classification for Participant 1 is higher than valence at $98.7 \%$. Also, higher than valence, the accuracy of classifying emotion based on arousal for Participant 2 is $99.8 \%$. However, most of the outputs with positive arousal are overlapped. For Participant 3, accuracy of the classification based on arousal is lower than valence. That is $74.9 \%$. Similar with valence, the classification based on arousal for Participant 4 is also about $64 \%$, where most of the instances are classified as positive arousal. With 16 interrupted segments, the accuracy of classifying arousal for Participant 5 is also around 50\%. The classification of arousal for Participant 6 is also around $99 \%$, similar to valence.

Thus, at average, the classification accuracy of valence for 6 participants is $79 \%$ and the classification accuracy of arousal is $81.1 \%$.

\subsection{Emotion classification of interpolated data}

Figure 6 shows the distribution of output on emotion classification using interpolated EEG data. The accuracy of valence and arousal classifications using interpolated data are similar to the classifications of interrupted data which are $97 \%$ and $98.7 \%$, respectively. For participant 2, the classification of valence improves to $97.7 \%$ for interpolated input, where the accuracy of classifying arousal remains around $99.8 \%$. However, the distribution of outputs with positive arousal are clearly dichotomized between the positive and negative valence. Distribution of outputs for Participant 3 using interpolated data has also improved with the accuracy of classifying valence at $88.6 \%$, and the accuracy of classifying arousal at $91.3 \%$.

Using interpolated data on 13 interrupted segments from Participant 4, the accuracy of classifying valence has improved from around $64 \%$ to $94 \%$. Likewise, the accuracy of arousal classification has also improved to $93 \%$. For Participant 5, the accuracy of classifying interpolated data has also improved from $50 \%$ to $89 \%$ and from $50 \%$ to $92 \%$ for valence and arousal, respectively. Interpolation is not implemented on the data from Participant 6 because it is already recorded in one segment.

Thus, in the average, the classification accuracy of valence for 6 participants is $94.2 \%$ and the classification accuracy of arousal is $95.6 \%$. 

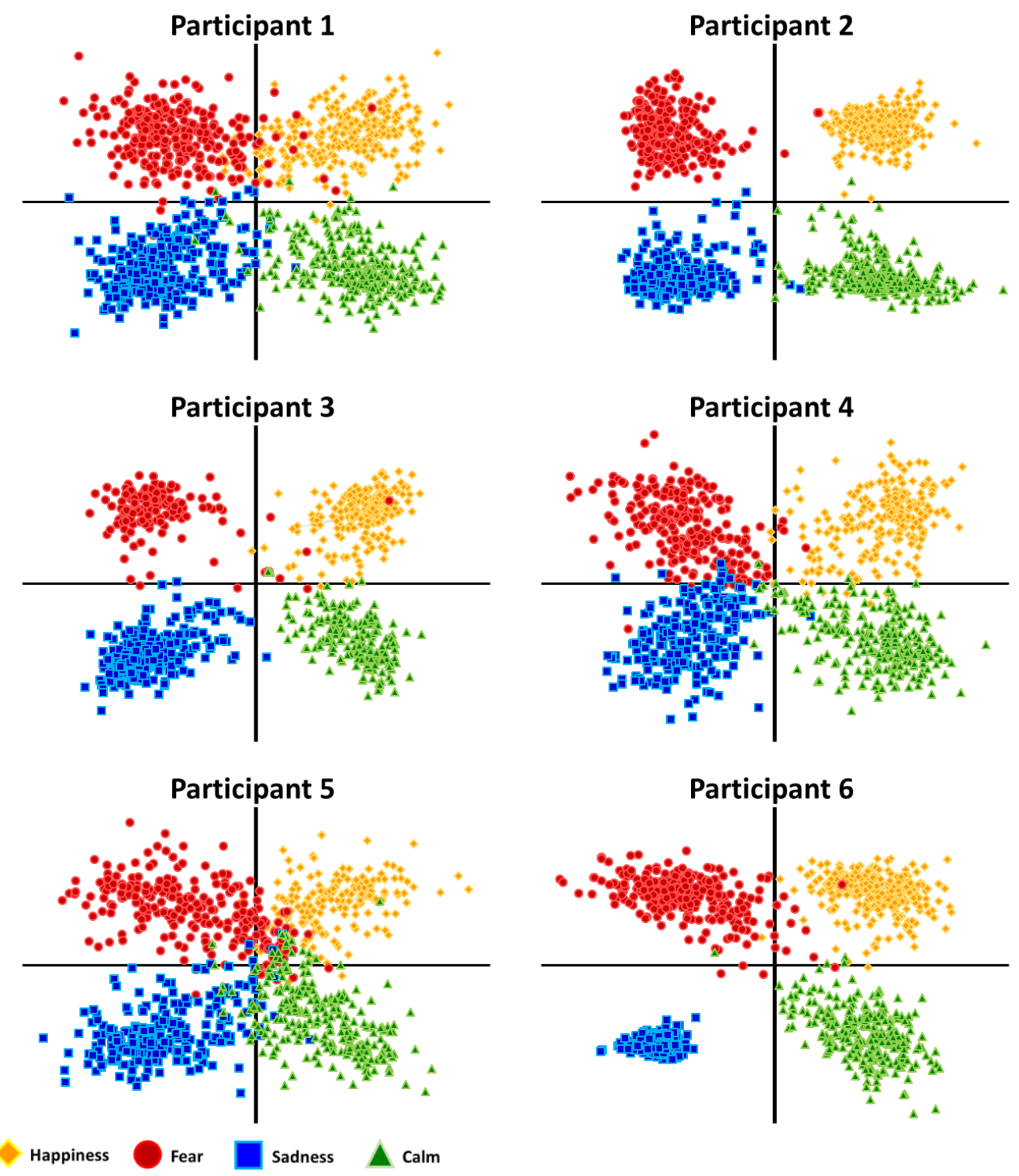

Figure 6 Output of MLP using interpolated EEG data on affective space model

\section{Conclusion}

This paper compares the results of profiling emotional states using MLP on originally interrupted EEG signals and corrected signals through interpolation. Based on the results, lower emotion classification accuracy is obtained from interrupted EEG signals, as the number of interrupted segments increases. This is represented by poor distribution of instances classification on a valence and arousal plot. It is also observed that, every time the signals are interrupted noise is captured and recorded.

Thus, in this paper, a technique is proposed to improve the accuracy of emotion profiling on interrupted EEG signals. The technique involves the suppression of noise regions in originally interrupted signals by no signal. This is done by marking the noise regions as no signal. Then, interpolation is performed on the suppressed sections.

As a result, emotion classification using MLP on interpolated data has shown that the accuracy has improved in all cases where the number of segments, variations from 1 segment to 19 segments for 400 seconds EEG recording. With that, proposed technique will be useful for performing classification through supervised learning on interrupted EEG. With the emergence of wireless EEG, more 
applications of processing interrupted signals are expected to take place.

\section{Acknowledgment}

This paper is supported by a Fundamental Research Grant Scheme (FRGS) funded by the Ministry of Higher Education (Grant code: FRGS16-029-0528).

\section{Conflicts of interest}

The authors have no conflicts of interest to declare.

\section{References}

[1] Yuen CT, San San W, Ho JH, Rizon M. Effectiveness of statistical features for human emotions classification using EEG biosensors. Research Journal of Applied Sciences, Engineering and Technology. 2013; 5(21):5083-9.

[2] Mampusti ET, Ng JS, Quinto JJ, Teng GL, Suarez MT, Trogo RS. Measuring academic affective states of students via brainwave signals. In third international conference on knowledge and systems engineering 2011 (pp. 226-31). IEEE.

[3] Othman M, Wahab A, Karim I, Dzulkifli MA, Alshaikli IF. EEG emotion recognition based on the dimensional models of emotions. Procedia-Social and Behavioral Sciences. 2013; 97:30-7.

[4] Rahnuma KS, Wahab A, Kamaruddin N, Majid H. EEG analysis for understanding stress based on affective model basis function. In 15th international symposium on consumer electronics (ISCE) 2011 (pp. 592-7). IEEE.

[5] Zhang Q, Lee M. Emotion development system by interacting with human EEG and natural scene understanding. Cognitive Systems Research. 2012; 14(1):37-49.

[6] Yaacob H, Wahab A. Affective state classification through CMAC-based model of affects (CCMA) using SVM. Advanced Science Letters. 2017; 23(11):1136973.

[7] Mert A, Akan A. Emotion recognition from EEG signals by using multivariate empirical mode decomposition. Pattern Analysis and Applications. 2018; 21(1):81-9.

[8] Liu S, Tong J, Meng J, Yang J, Zhao X, He F, et al. Study on an effective cross-stimulus emotion recognition model using EEGs based on feature selection and support vector machine. International Journal of Machine Learning and Cybernetics. 2018; 9(5):721-6.

[9] Soleymani M, Pantic M, Pun T. Multimodal emotion recognition in response to videos. IEEE Transactions on Affective Computing. 2012; 3(2):211-23.

[10] Yoon HJ, Chung SY. EEG-based emotion estimation using Bayesian weighted-log-posterior function and perceptron convergence algorithm. Computers in Biology and Medicine. 2013; 43(12):2230-7.

[11] Nie D, Wang XW, Shi LC, Lu BL. EEG-based emotion recognition during watching movies. In 5th international IEEE/EMBS conference on neural engineering 2011 (pp. 667-70). IEEE.

[12] Nor NM, Salleh SH, Zubaidi A. Understanding teacher stress when teaching the developed technology by using electroencephalogram (EEG) signals. Journal of Applied and Physical Sciences. 2016; 2(3):65-76.

[13] Bhatti AM, Majid M, Anwar SM, Khan B. Human emotion recognition and analysis in response to audio music using brain signals. Computers in Human Behavior. 2016; 65:267-75.

[14] Murugappan M, Juhari MR, Nagarajan R, Yaacob S. An investigation on visual and audiovisual stimulus based emotion recognition using EEG. International Journal of Medical Engineering and Informatics. 2009; 1(3):342-56.

[15] Liu YJ, Yu M, Zhao G, Song J, Ge Y, Shi Y. Realtime movie-induced discrete emotion recognition from EEG signals. IEEE Transactions on Affective Computing. 2018; 9(4):550-62.

[16] Bastos-Filho TF, Ferreira A, Atencio AC, Arjunan S, Kumar D. Evaluation of feature extraction techniques in emotional state recognition. In 4th international conference on intelligent human computer interaction (IHCI) 2012 (pp. 1-6). IEEE.

[17] Mutasim AK, Tipu RS, Bashar MR, Amin MA. Video category classification using wireless EEG. In international conference on brain informatics 2017 (pp. 39-48). Springer, Cham.

[18] Song T, Zheng W, Song P, Cui Z. EEG emotion recognition using dynamical graph convolutional neural networks. IEEE Transactions on Affective Computing. 2018.

[19] Alarcao SM, Fonseca MJ. Emotions recognition using EEG signals: a survey. IEEE Transactions on Affective Computing. 2017.

[20] Lang PJ. International affective picture system (IAPS): affective ratings of pictures and instruction manual. Technical Report. 2005.

[21] Liu YH, Wu CT, Kao YH, Chen YT. Single-trial EEG-based emotion recognition using kernel Eigenemotion pattern and adaptive support vector machine. In annual international conference of the engineering in medicine and biology society (EMBC) $2013 \mathrm{Jul} 3$ (pp. 4306-9). IEEE.

[22] Khalili Z, Moradi MH. Emotion detection using brain and peripheral signals. In cairo international biomedical engineering conference 2008 (pp. 1-4). IEEE.

[23] Hosseini SA, Khalilzadeh MA. Emotional stress recognition system using EEG and psychophysiological signals: using new labelling process of EEG signals in emotional stress state. In international conference on biomedical engineering and computer science 2010 (pp. 1-6). IEEE.

[24] Hosseini SA, Naghibi-Sistani MB. Emotion recognition method using entropy analysis of EEG signals. International Journal of Image, Graphics and Signal Processing. 2011; 3(5):30-6.

[25] Chanel G, Kronegg J, Grandjean D, Pun T. Emotion assessment: Arousal evaluation using EEG's and 
peripheral physiological signals. In international workshop on multimedia content representation, classification and security 2006 (pp. 530-7). Springer, Berlin, Heidelberg.

[26] Lee YY, Hsieh S. Classifying different emotional states by means of EEG-based functional connectivity patterns. PloS one. 2014; 9(4):e95415.

[27] Handayani D, Wahab A, Yaacob H. Evaluation of feature extraction and classification techniques for EEG-based subject identification. Jurnal Teknologi. 2016; 78(9-3):41-8.

[28] Nakisa B, Rastgoo MN, Tjondronegoro D, Chandran V. Evolutionary computation algorithms for feature selection of EEG-based emotion recognition using mobile sensors. Expert Systems with Applications. 2018; 93:143-55

[29] Semmlow J. Signals and systems for bioengineers: a MATLAB-based introduction. Academic Press; 2011.

[30] Niedermeyer E, da Silva FL. Electroencephalography: basic principles, clinical applications, and related fields. Lippincott Williams \& Wilkins; 2005.

[31] Russell JA. A circumplex model of affect. Journal of Personality and Social Psychology. 1980; 39(6):116178.

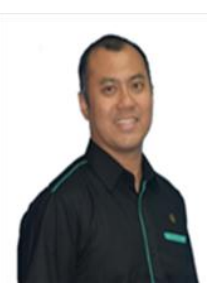

Hamwira Yaacob is an Assistant Professor at International Islamic University Malaysia (IIUM). He has great interests in computational intelligence and Neurocomputing. His doctoral thesis on Emotion Profiling using CMAC-based Computational Models of Affects marks the development of his expertise in an area that consolidates Computational Intelligence, Human Affective States, and Brain Cognitive Studies. Most of his studies involve research works which aim to understand human behaviours based on the dynamics of various affective state stimulations that are manifested and captured from activated brain electrical signals. Since 2006, he has been teaching several subjects in Computer Science, including Discrete Mathematics, Data Structures \& Algorithms and Java Programming.

Email: hyaacob@iium.edu.my

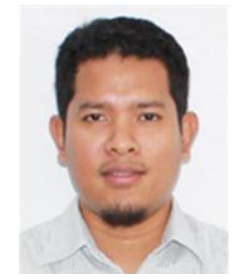

Hazim Omar is a Science Officer at Hospital Universiti Sains Malaysia (HUSM). He has an acute and broad interest in physics and its application which mainly focuses on computational of signal processing, imaging modalities and its approach techniques as well as understanding the principle behind human brain processing. Receiving his master degree in Translational Neuroimaging (2012) by working with the computational and mathematical part of brain functional modelling to detect the so called "small-world" architectures in resting state network. Currently he is pursuing his $\mathrm{PhD}$ at USM exploring the Brain Connectivity Characteristics from Traumatic Brain Injury data obtained from different neuroimaging modalities which are EEG, MEG and fMRI.

Email: hazim@usm.my

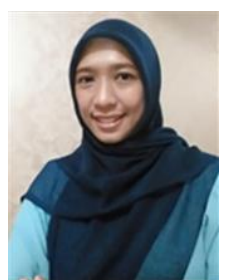

Dini Handayani is a lecturer at Taylo's University, Malaysia. She received her degree in Informatics Engineering in 2002, Master of Information Technology degree in 2011 and $\mathrm{PhD}$ in Computer Science in 2017 at the International Islamic University Malaysia (IIUM). Prior to that, she worked several years as a Software Developer. She is currently involved in the several research projects where she pursues her research interests in the area of Affective Computing and Computational Intelligence with a focus on Emotion and Mood Profiling.

Email: diniOktarina.DwiHandayani@ taylors.edu.my

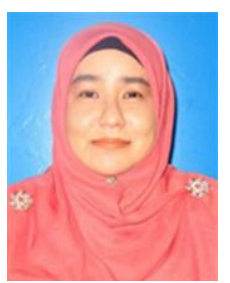

Raini Hassan is an Assistant Professor at the Department of Computer Science, Kulliyyah of Information and Communication Technology (KICT), International Islamic University Malaysia (IIUM). She has been working in the area of Artificial Intelligence since 2003 where she did a Master dissertation on fuzzy logic. Her PhD work was on feature selection for Evolving Connectionist Systems (ECOS), and this is where she got a little exposure on big data. Her main research interests are data analytics and the application of machine learning in data science, big data, and Neurocomputing; where currently she is working with event-related potential (ERP) brain signals for health profiling. She has experience in teaching the following undergraduate computer science courses: Computer Organization, $\mathrm{C}++$, Principles of Artificial Intelligence, Probability and Statistics, Data Science, Big Data Analytics, and a master course entitled Unstructured Data Analytics.

Email: hrai@iium.edu.my 